\title{
الازدواج اللغوي في الكتابة الأدبية: فضاء " المابين "
}

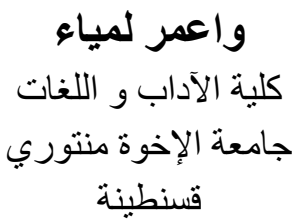

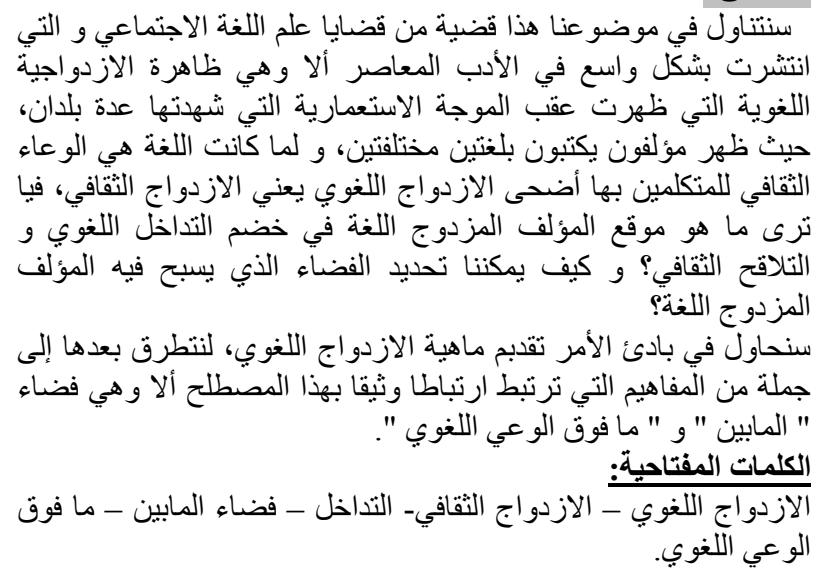

\section{مقدمة: Résumé:}

مما لا جدال فيه أنه لا بمكنتا

Le présent article a pour sujet un phénomène linguistique qui a fait un grand écho et qui est devenu une tendance dans le domaine de la littérature moderne qui est le « Bilinguisme».

Le bilinguisme a émergé suite au mouvement colonial qui a touché plusieurs pays, les écrivains ont commencé à écrire en deux langues différentes celle de leur pays natal et celle du colonialiste donnant ainsi naissance à une interférence entre deux cultures différentes, et qui dit bilinguisme dit biculturalisme. Nous allons essayer par le présent travail de répondre à certaines questions qui se posent et qui s'imposent à propos du bilinguisme à savoir : dans quelle position se situe l'auteur bilingue au sein de l'interférence linguistique et culturelle? Et comment pourra- t- on définir l'espace dans lequel plonge l'auteur bilingue?

D'abord, nous allons définir la notion du «Bilinguisme», puis nous allons aborder certains concepts qui sont intimement liés à cette notion à l'instar de " l'Entre-deux mondes" et « la surconscience linguistique $»$.

\section{Mots clés:}

Bilinguisme - Biculturalisme- l'interférence- l'entre-deux mondesla surconscience linguistique. 
على القارئ المتلقي للنتاج الأدبي ـالذي يكتبه مؤلف مزدوج اللغةــ حيث قد يشعر بنوع من الغر ابة لدى قر ائته للعمل الأدبي.

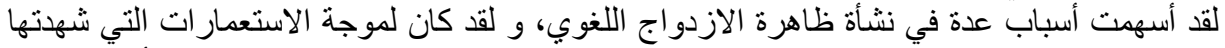

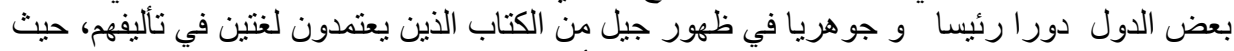

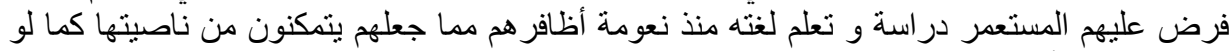

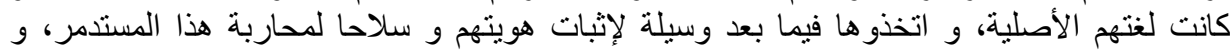

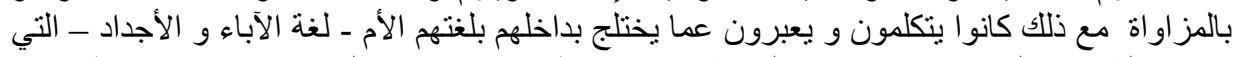

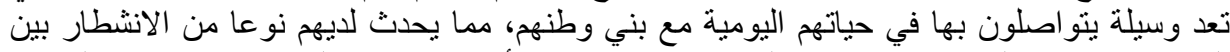

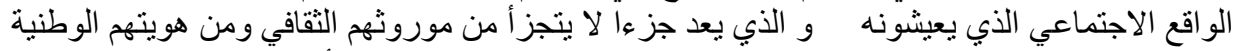

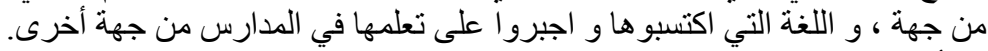

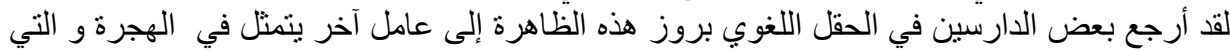

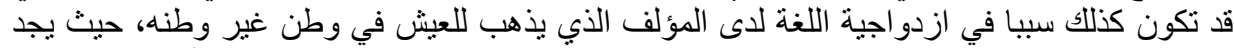

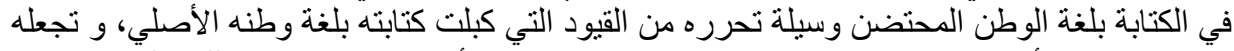

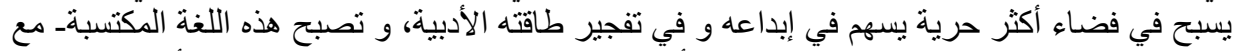

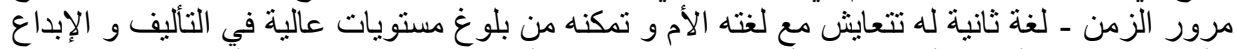

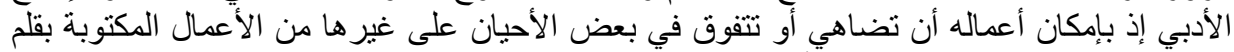

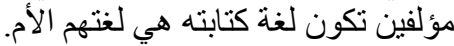

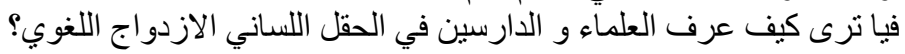

\section{1 - مفهوم الازدواج اللغوي:}

لقد حظي مفهوم الازدواج اللغوي بتعريفات عدة منذ الإدون القديم، إذ مع نهاية العصور الوسطى و بداية

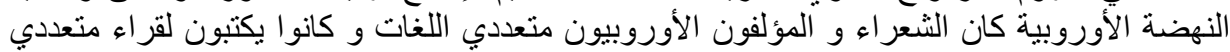

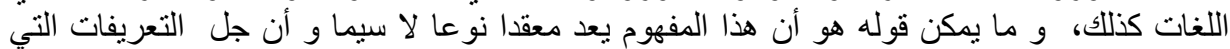

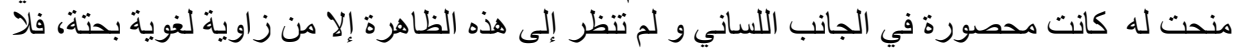

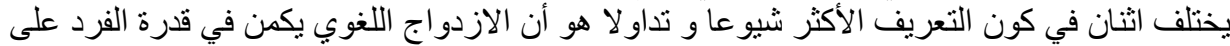

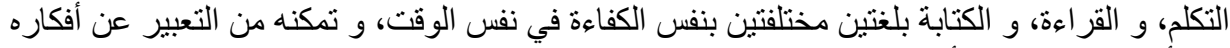
بلغة أخرى غير لغته الأم. لقد عرف المختص في علم النفس و الباحث رانزو تيتون Renzo Titone الازدواج اللغون التوي على أنه

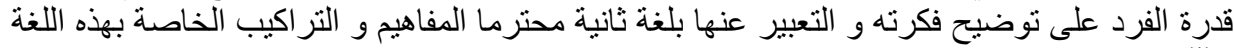

حسب هذا التعريف، يظهر الازدواج اللغوي على أنه تمكن الفرد من ناصية لغة ثانية إلى جانب لغته الإنه

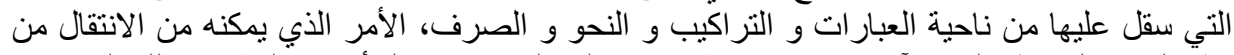

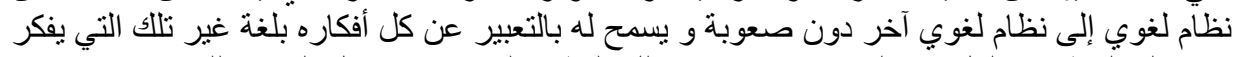

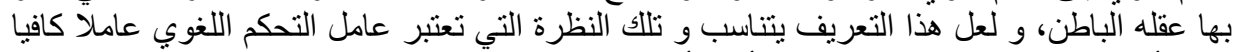

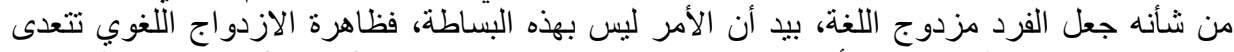

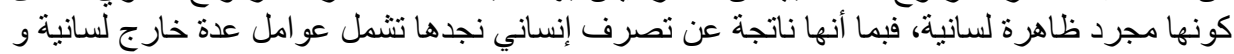

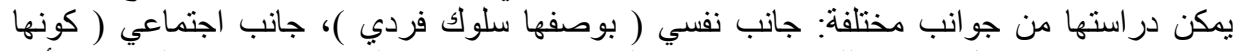

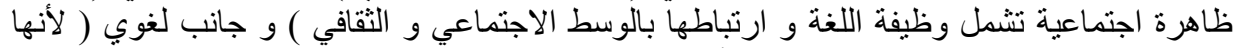

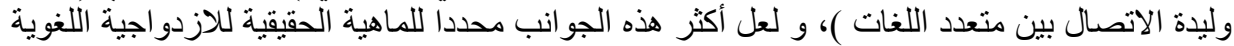

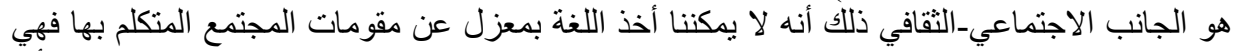

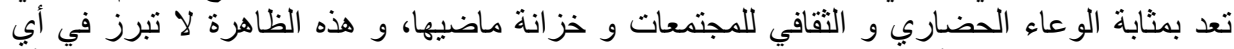

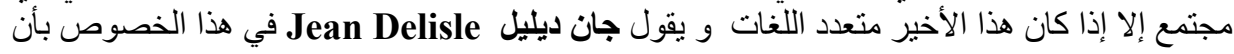


الازدو اج اللغوي ظاهرة معقدة ليس من السهل رسم حدودها، إذ هنالك قرابة تجمع الازدواج اللغوي

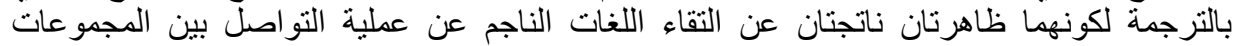

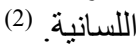

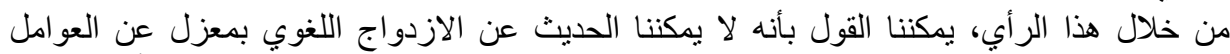

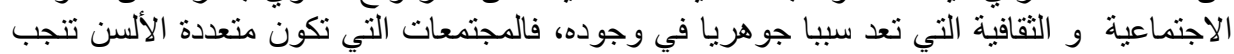

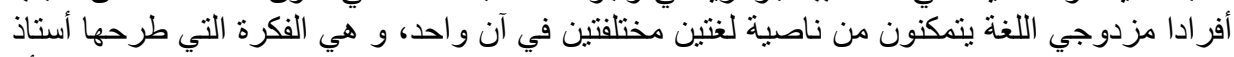

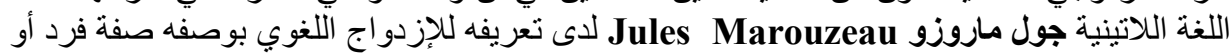
جماعة يستعملون لغتين بطلاقة دون أن يكون هناك فرق ملحوظ في اتقانهما للغة على حساب الأخرى.

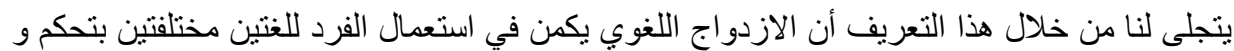

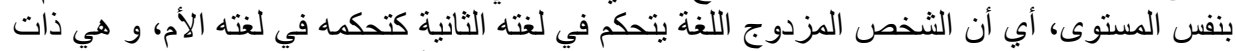

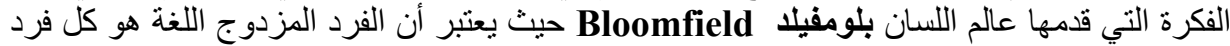

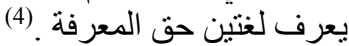

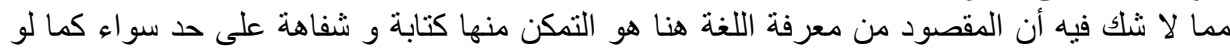

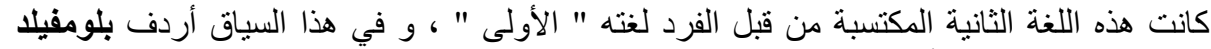

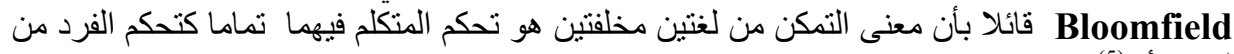

غير أن الأمر في الحقيقة لبس كذلك، فمهما بلغ تمكن الفرد من لغة ثانية إلى جانب لغته الأم إلا أنه من

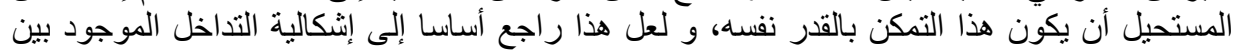

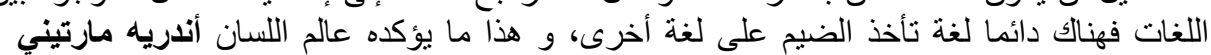
André Martinet

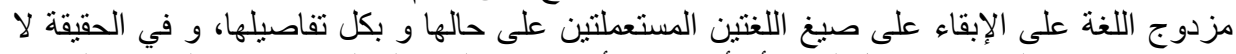

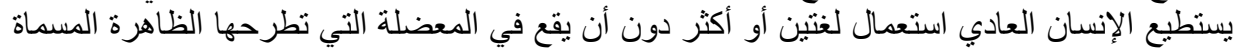

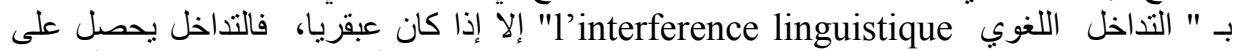

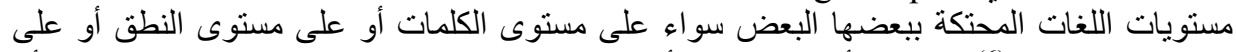

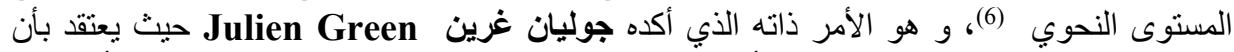

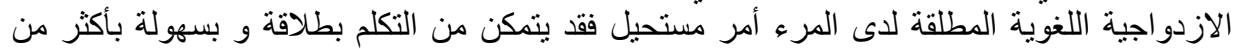

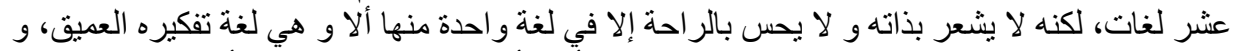

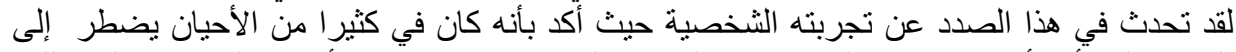
التفكير بلغة أو بأخرى بحسب الظروف، و لكن في لحظات درامية كانت أفكاره العميقة تتجلى باللغة التئ

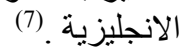

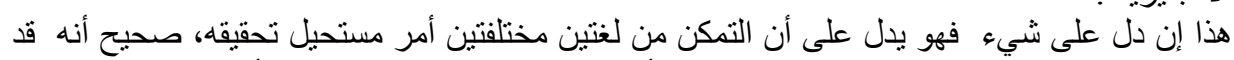

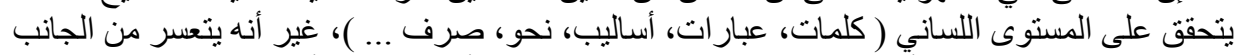

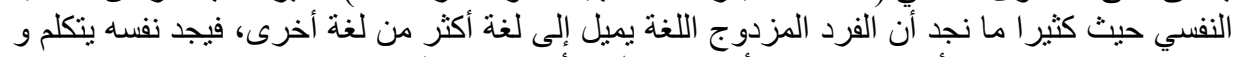

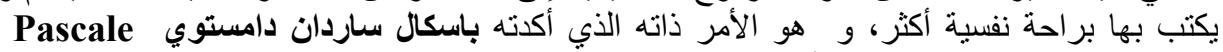
Sardin Damestoy

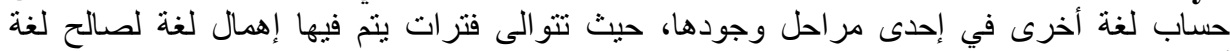

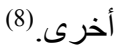

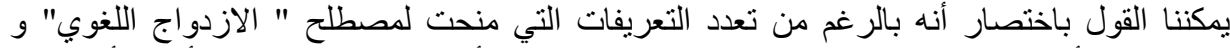

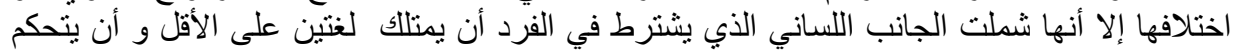

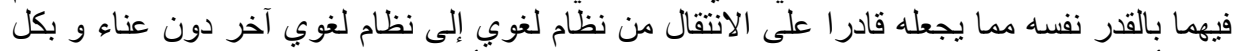

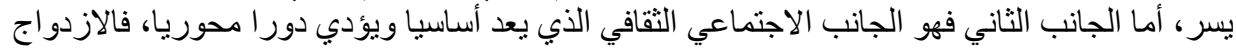


اللغوي يعني حتما الازدواج النقافي، ذلك أن اللغة هي المر آة التي تنعكس من خلالها ثقافة المتكلمين بها

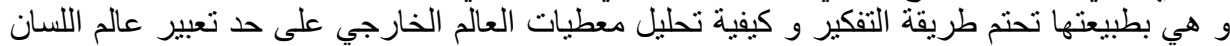

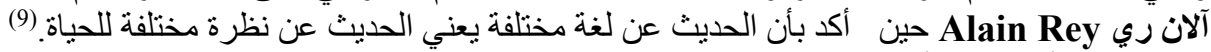

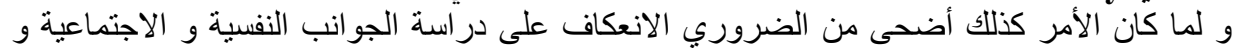

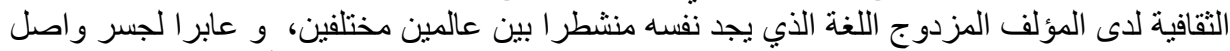

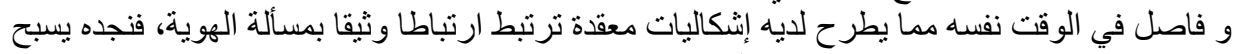

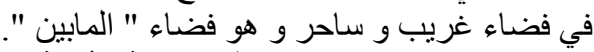

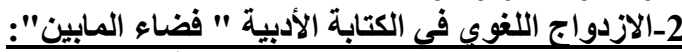

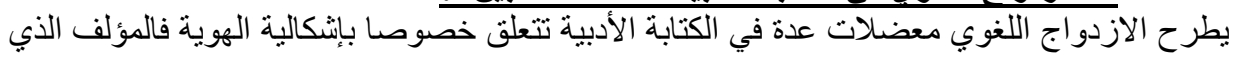

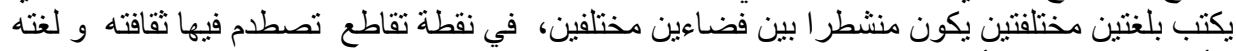

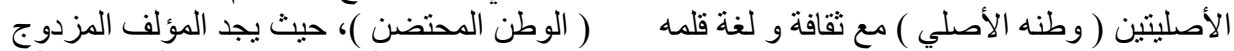

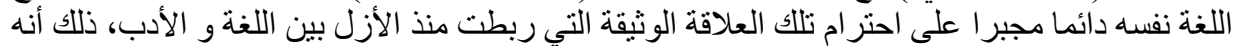

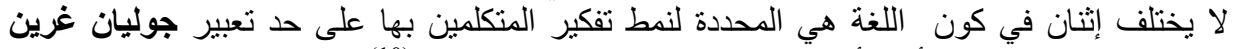
Green Julien

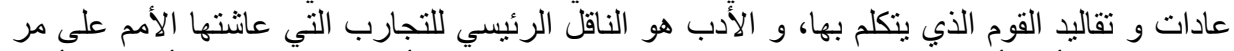

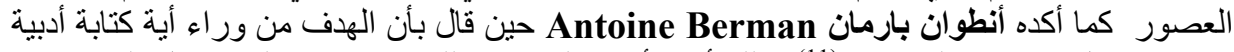

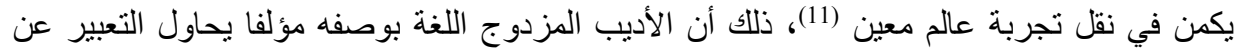

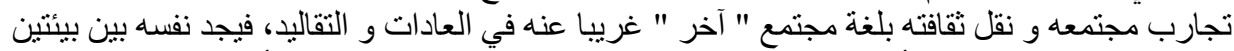

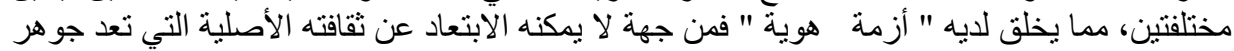

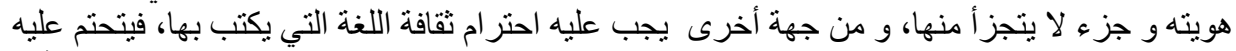

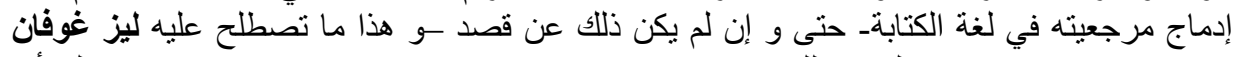

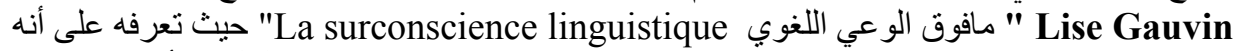

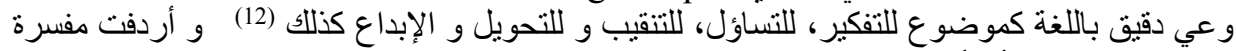

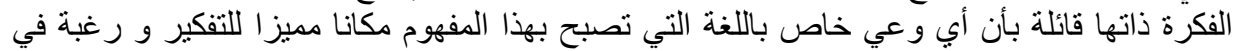

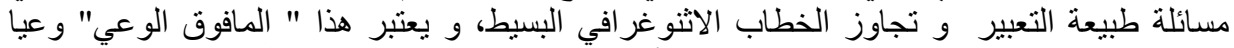

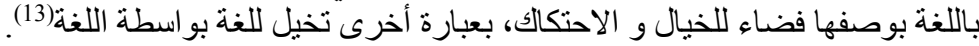

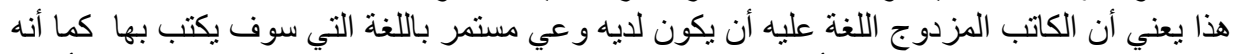

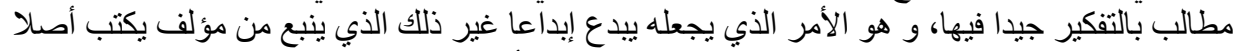

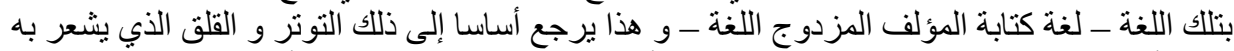

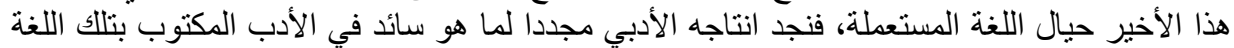

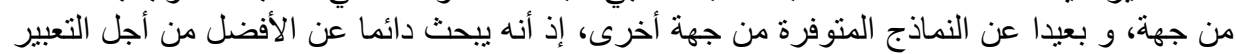

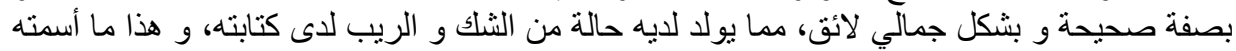

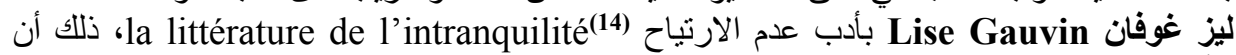

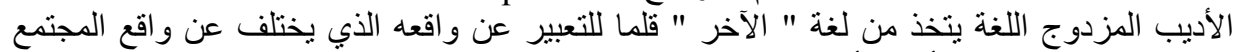

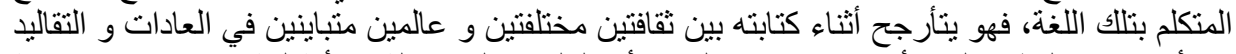

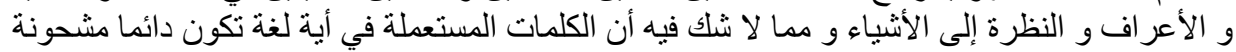

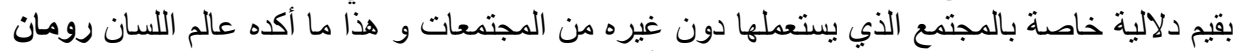

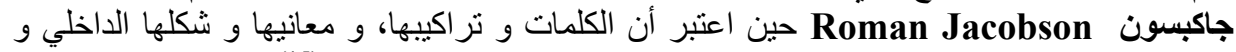

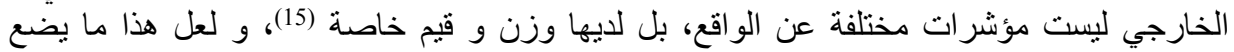

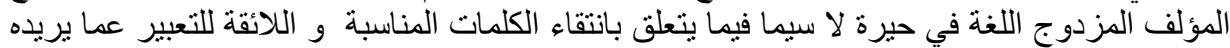

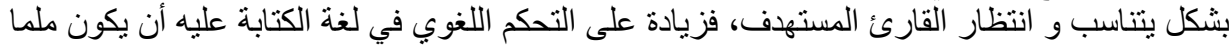
كذللك بثقافة المجتمع الذي يتوجه إليه من خلال كتاباته على حد تعبير رانزو تيتون Renzo Titone 
حين قال بأن الفرد المزدوج اللغة لا يتحكم في المجال البنيوي لنظامين لغويين فحسب، بل أكثر بكثير

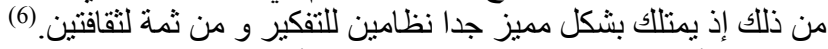

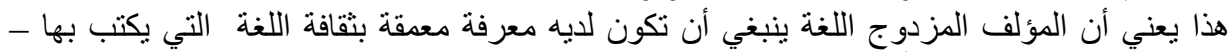

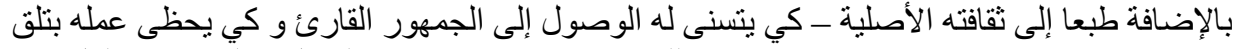

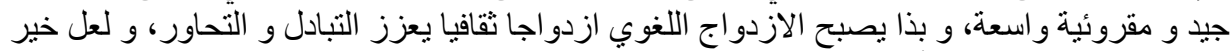

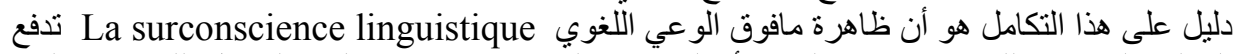

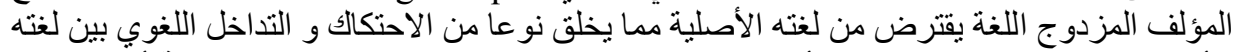

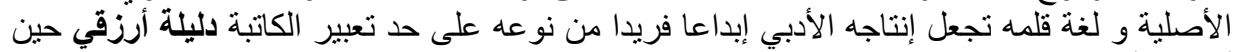

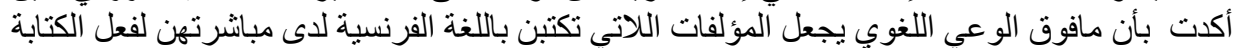

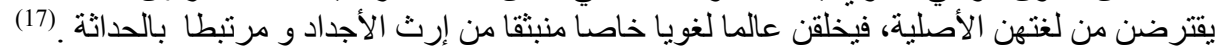

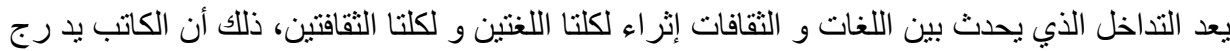

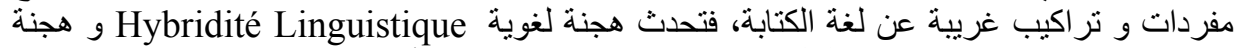
ثقافية Hybridité Culturelle

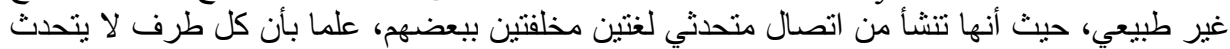

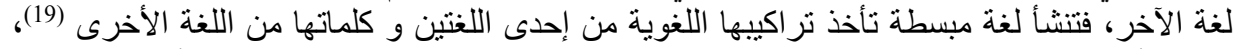

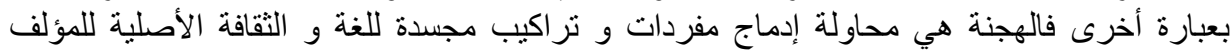

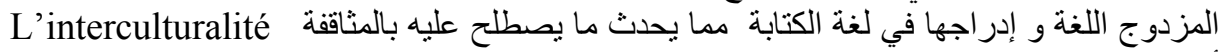

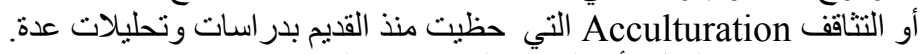

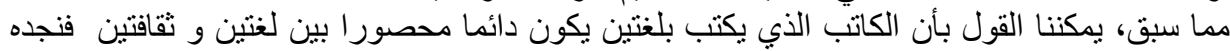

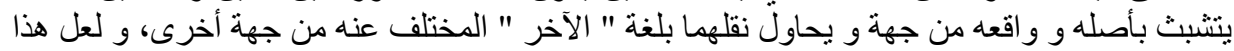

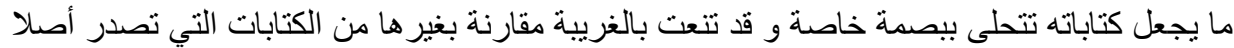

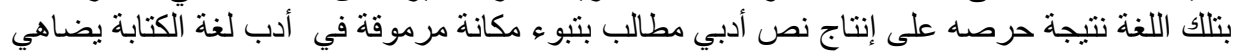

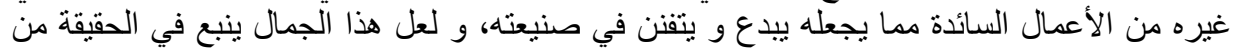

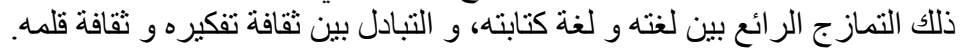

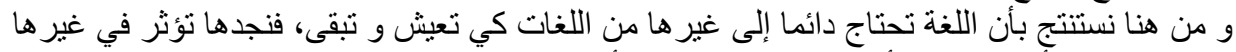

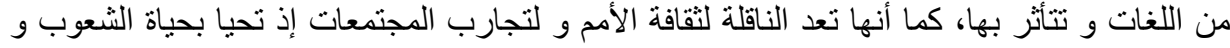

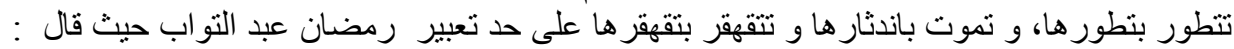

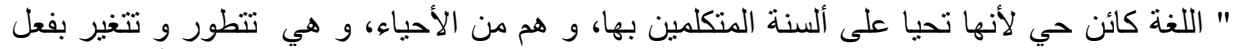

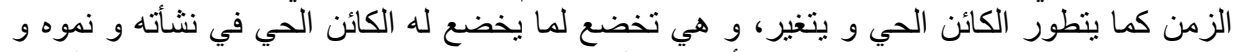

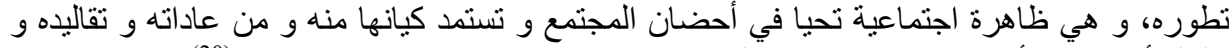

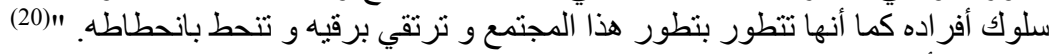

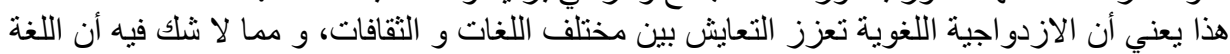

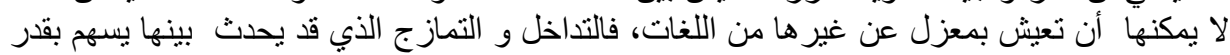

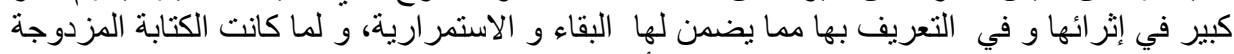

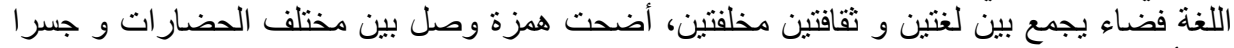

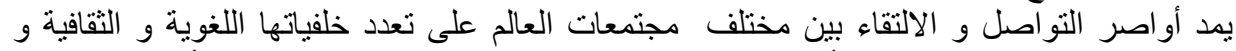
الاجتماعية و تصبح بذا عاملا أساسا في التطور اللغوي و في الحوار الثقافي فاتحة أبواب التبادل و و

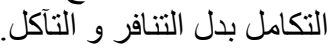




$$
\text { قائمة المر اجع و المصادر: }
$$

- André Martinet, Eléments de linguistique générale, Armand Collin, Paris, 1980.

- Antoine Berman, la traduction et la lettre ou l'Auberge du lointain, Seuil, Paris, novembre 1999.

- Arezki Dalila, Romancières algériennes francophones : langue, culture, identité ( Biarritz : Atlantica-Séguier ), 2005.

- Jean Delisle, L'analyse du discours comme méthode de traduction, presses de l'université d'Ottawa, 1984.

- Julien Green, le langage et son double, Seuil, Paris, 1987.

- Lise Gauvin, La fabrique de la langue, Seuil, Paris, 2004,

- Lise Gauvin, Langagement : l'écrivain et la langue au Québec, revue internationale d'études Québécoises, Montréal, Boréal, 2000.

- Lise Gauvin, Autour du concept de littérature mineure, variation sur un thème majeure, in Jean Pierre Bertrand, Lise Gauvin, Laurent Demoulin ( éd ), littératures mineures en langues majeures, presses universitaires de Montréal, 2003.

- Pascale Sardin Damestoy, samuel Beckett auto-traducteur ou 1'art de l'empêchement, Artois presse université, 2002.

- Renzo Titone, Le bilinguisme précoce, Armando Armando, Rome, 1972.

- Recueil d'articles et d'études sur les avantages d'une éducation bilingue ou plurilingue précoce, consulté sur le lien électronique : multilingues-précoces.over-blog.com le 22/03/2017 à 17.00.

- Silvia Audo Gianotti, A Diary, le journal de Julien Green entre bilinguisme et auto-traduction, Aux frontières de la culture et de la langue, textes édités sous la direction de Christian Lagarde et Helena Tanquiero, Lambert Lucas, France, janvier 2014.

\footnotetext{
الهو امش:

${ }^{1}$ Voir Renzo Titone, Le Bilinguisme Précoce, Armando Armando, Rome, 1972, P.11.

${ }^{2}$ Jean Delisle, L'analyse du discours comme méthode de traduction ( Paris I ), Presses de l'université d'Ottawa, 1984, P.34

${ }^{3}$ Voir Renzo Titone, Op.cit, P.12.

${ }^{4}$ Silvia Audo Gianotti : A Diary, le journal de Julien Green entre bilinguisme et auto-traduction, Aux frontières de la culture et de la langue, textes édités sous
} 
la direction de Christian Lagarde et Helena Tanquiero, Lambert-Lucas, Janvier 2014, France, P.80.

${ }^{5}$ Ibid.

${ }^{6}$ Voir André Martinet, Eléments de linguistique générale, Armand Collin, Paris,1980, pp.169-170.

${ }^{7}$ Julien Green, Le langage et son double, Editions du Seuil, Paris, 1987, P.167.

${ }^{8}$ Pascale Sardin Damestoy, Samuel Beckett auto-traducteur ou l'art de l'empêchement, Artois Presse Université, 2002, P. 25.

${ }^{9}$ Recueil d'articles et d'études sur les avantages d'une éducation bilingue ou plurilingue précoce, consulté sur le lien électronique : multilinguesprécoces.over-blog.com le 22/03/2017 à 17.00.

${ }^{10}$ Julien Green, Le langage et son double, Editions du Seuil, Paris, 1987, P.213.

${ }^{11}$ Antoine Berman, la traduction et la lettre ou l'Auberge du lointain, Seuil, Paris, novembre 1999, P.70.

${ }^{12}$ Lise Gauvin, Langagement : l'écrivain et la langue au Québec, revue internationale d'études Québécoises, Montréal, Boréal, 2000, P.209.

${ }^{13}$ Lise Gauvin, Autour du concept de littérature mineure, variation sur un thème majeure, in Jean Pierre Bertrand, Lise Gauvin, Laurent Demoulin ( éd ), littératures mineures en langues majeures, presses universitaires de Montréal, 2003 , P.19.

${ }^{14}$ Lise Gauvin, La fabrique de la langue, Seuil, Paris, 2004, P.259.

${ }^{15}$ Pascale Sardin Damestoy, Samuel Beckett auto-traducteur ou l'art de l'empêchement, Artois Press Université, 2002, P. 42

${ }^{16}$ Renzo Titone, Le Bilinguisme Précoce, Armando Armando, Rome, 1972, P.05.

${ }^{17}$ Arezki Dalila, Romancières algériennes francophones : langue, culture, identité ( Biarritz : Atlantica-Séguier, 2005), P.29.

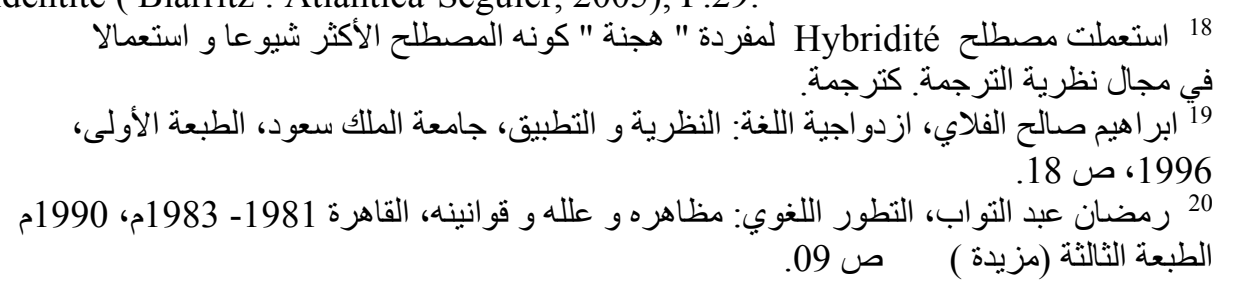

